\title{
Doença de além-mar: estudos comparativos da oncocercose na América Latina e África
}

\author{
Overseas disease: comparative \\ studies of oncocercosis in \\ Latin America and Africa
}

\author{
Magali Romero Sá \\ Pesquisadora da Casa de Oswaldo Cruz \\ Av. Brasil, 4365 21045-900 Rio de Janeiro - RJ Brasil \\ magali@coc.fiocruz.br \\ Marilza Maia-Herzog \\ Pesquisadora titular do Departamento de Entomologia/IOC/Fiocruz \\ Doutora em biologia parasitária \\ Av. Brasil, 4365 21045-900 Rio de Janeiro - RJ Brasil \\ mherzog@ioc.fiocruz.br
}

\begin{abstract}
A oncocercose é causada por um verme parasita, a Onchocerca, e transmitida pelo mosquito borrachudo, simuliídeo, podendo causar cegueira em seu estado agudo. Oriunda do continente africano onde é disseminada, foi descoberta na América Latina, na Guatemala, em 1917, com registros posteriores no México, Venezuela, Colômbia e Brasil. O estabelecimento da doença nas Américas tem intrigado os especialistas, e ainda hoje é uma questão em aberto. O projeto multidisciplinar descrito nesta nota de pesquisa objetiva investigar os aspectos históricos da introdução e disseminação da doença na América Latina, e realizar estudos comparativos sobre a história da doença nos dois continentes. A importância que adquiriu acarretou a revalorização das coleções de simuliídeos, cujas espécies brasileiras começaram a ser estudadas por Adolpho Lutz no início do século XX.
\end{abstract}

PALAVRAS-CHAVE: oncocercose, índios ianomâmi, relações Brasil e África, mosquitos, vermes.

Round worm is a parasite, Onchocerca, and is transmitted by a black fly, simuliideo; it can cause blindness. Originally from the African continent, where it is widespread, in Latin America it was first discovered in Guatemala in 1917; later instances were recorded in Mexico, Venezuela, Colombia and Brazil. The establishment of this disease in the Americas has intrigued scientists since then and today it is an open question. The multidisciplinary project described in this research note aims to investigate historical aspects of the arrival and spread of the disease in Latin America and, to make comparative studies of the history of the disease on both continents. The increasing importance of the disease has meant greater value being attributed to collections of simuliideo, whose Brazilian species were first studied by Adolpho Lutz at the beginning of the $20^{\text {th }}$ century.

KEYWORDS: oncocercosis, Ianomami indians, relations between Brazil and Africa, mosquitos, worms. 
$\mathrm{O}$ presente projeto originou-se de pesquisa iniciada em 2000 em associação com a pesquisadora Marilza Maia-Herzog, do Laboratório de Simuliídeos do Instituto Oswaldo Cruz (IOC). Representa um segmento de Coleções Científicas da Fundação Oswaldo Cruz, linha de pesquisa que incluiu projetos como 'Biological Collections as Archives of Basic Data for Applied Health Research in Tropical Regions', em conjunto com a Wellcome Unit for the History of Medicine na University of Oxford ${ }^{1} \mathrm{e}$ 'Adolpho Lutz e a história da medicina tropical no Brasil,' em desenvolvimento. ${ }^{2}$

A oncocercose é uma doença transmitida por um verme parasita que causa cegueira. Foi descoberta, primeiro, na África em $1875,{ }^{3}$ mas seu modo de transmissão só foi desvendado mais de cinqüenta anos depois, em 1926. Comprovou-se, então, que o vetor era um diminuto e agressivo díptera (simuliídeo), conhecido vulgarmente no Brasil como piúm ou borrachudo. ${ }^{4}$ Endêmica no continente africano, a oncocercose teve seu primeiro caso registrado no Novo Mundo em 1915, na Guatemala. Em 1923, foi detectada no México; em 1949, na Venezuela; em 1965, na Colômbia; e, finalmente, em 1967, no Brasil, na fronteira com a Venezuela.

Hipóteses têm sido propostas para explicar o estabelecimento da doença nas Américas e sua forma de dispersão, todas, porém, sem comprovação.

O presente projeto tem por objetivo investigar os aspectos históricos relacionados à introdução e disseminação da doença na América Latina, e analisar comparativamente os resultados com a história da evolução da doença no continente africano.

\section{Documento de descrição detalhada}

Em fins do século XIX e início do XX, os estudos sobre parasitos e seus vetores/hospedeiros estavam em pleno desenvolvimento. Com a disseminação da teoria microbiana e a descoberta dos agentes etiológicos

\footnotetext{
${ }^{1}$ Projeto coordenado no Brasil por Magali Romero Sá e Jaime L. Benchimol, e, na Inglaterra, por Nancy Stepan e David Knight, com apoio do British Council e da Fundação Oswaldo Cruz. Período: 1988-2000.

${ }^{2}$ Projeto financiado pelo Conselho Nacional de Desenvolvimento Científico e Tecnológico (CNPq), Fundação de Amparo à Pesquisa do Rio de Janeiro (FAPERJ), Fundação Oswaldo Cruz e Instituto Adolfo Lutz.

${ }^{3}$ A oncocercose humana é causada por um verme parasita e pode levar à cegueira. Os vermes adultos são encontrados enrodilhados no interior de tumores fibrosos, subcutâneos, chamados oncocercomas. Ao se reproduzirem, produzem um grande número de microfilárias, que migram no tecido subcutâneo e são, então, ingeridas por insetos hematófagos (simuliídeos). Os típicos nódulos podem ser encontrados em qualquer parte do corpo, sendo, porém, mais freqüentes perto das articulações e na cabeça.

${ }^{4}$ Os simuliídeos pertencem ao grupo Diptera, família Simuliidae. Reconhecidos no passado devido à agressividade das fêmeas adultas, sabe-se hoje que são vetores de vírus, protozoários e vermes, causando sérios problemas à saúde do homem e de animais.
} 
de doenças como elefantíase, malária, peste bubônica e febre amarela, os países colonizadores saíram em busca desses agentes por meio de numerosas expedições exploratórias a países ditos tropicais. No Brasil, pesquisadores como Adolpho Lutz, Oswaldo Cruz, Arthur Neiva e Carlos Chagas, entre outros, coletavam, descreviam novas espécies, trocavam informações e espécimes com seus pares em diferentes países.

Até fins do século XIX a oncocercose era conhecida apenas da África, sendo que os estudiosos europeus tinham conhecimento de casos isolados naquele continente. Na virada do século XIX para o $\mathrm{XX}$, casos começaram a ser notificados em várias localidades, o que possibilitou um mapeamento mais preciso da incidência da doença. Expedições médico-científicas começaram a ser enviadas pelos países colonizadores. A Escola de Medicina Tropical de Liverpool, desde a sua fundação, em 1899, até o início da Primeira Guerra Mundial, em 1914, enviou 18 expedições à África com o objetivo de mapear suas condições de saúde, e constatou a alta incidência da oncocercose em Gana e Serra Leoa.

A busca do agente transmissor da doença intensificou-se. Em 1904, em excursão ao rio Uelé, o parasitologista francês Émile Brumpt chamou a atenção para a prevalência da doença entre aqueles que habitavam as suas margens e sugeriu a possibilidade de transmissão por algum inseto hematófago comum na área, o que foi comprovado depois.

Com o início da Primeira Guerra Mundial, as pesquisas sobre a oncocercose foram interrompidas, já que os problemas sanitários da África deixaram de ser prioridade. Não obstante essa letargia conjuntural, descoberta relevante na América Latina deixou os estudiosos da doença intrigados, suscitando novas questões em relação à origem e disseminação da doença no Novo Mundo. Em 1915, o médico guatemalteco Rodolfo Robles atendeu uma paciente com enrijecimento e inchaço na face e problemas oculares. Posteriormente, constatou que esse quadro clínico era comum na região costeira daquele país, sendo chamado pelos nativos de erisipela de la costa. Ao examinar o material do nódulo extirpado de um segundo paciente com os mesmos sintomas, Robles observou a presença de pequenos vermes, que associou aos distúrbios oculares comuns em pacientes que apresentavam aqueles nódulos subcutâneos. O parasitologista francês Émile Brumpt confirmou a identificação dos vermes como pertencentes ao gênero Onchocerca, o mesmo que causava a doença denominada craw craw pelos africanos. Robles enunciou a hipótese de que o transmissor deveria ser um inseto sugador de sangue, possivelmente um simuliídeo.

No Brasil, os simuliídeos começavam a ser investigados como possíveis transmissores de doenças. Em 1909, o médico e cientista Adolpho Lutz, dando continuidade a seus estudos sobre dípteros sugadores de sangue, publicou estudo sobre as espécies brasileiras de simuliídeos, no qual declarou não haver evidências de que seriam transmissores de doenças infecto-parasitárias. 
Após a descoberta de Robles, começaram a surgir no México casos similares à síndrome identificada na costa guatemalteca, com relatos de povoações inteiras com graves problemas oculares, inclusive cegueira. Focos foram encontrados nos estados de Oaxaca (serra de Ixtlán) e Chiapas (Soconusco e Chamula). Estudos subseqüentes demonstraram que a incidência da doença era maior em áreas florestadas de altitude e em regiões produtoras de café, sendo o sul de Chiapas uma área hiperendêmica, o que foi relacionado com os movimentos migratórios entre esta região e o outro foco endêmico, Huehuetenago, na Guatemala.

A preocupação com o avanço da oncocercose na América da Norte levou médicos do Departamento de Medicina Tropical da Universidade de Harvard a se interessarem pela doença, e empreenderem rigorosa investigação nas três Américas. Já em 1924-25, a Amazônia brasileira era visitada pela expedição de Hamilton Rice, médico e explorador norte-americano que realizou levantamentos geográficos e médicos na bacia do rio Branco até a nascente de seu afluente Uraricoera, na serra Parima. Os médicos da expedição realizaram extenso inventário das doenças locais e coletaram animais de vários grupos zoológicos potencialmente vetores ou transmissores de doenças. A oncocercose não foi localizada na região, muito embora simuliídeos tenham sido coletados e examinados àquela época. No relatório médico da expedição, lê-se:

o Simulium amazonicum é considerado uma peste em muitas partes da Amazônia. Conhecido localmente por piúm ou borrachudo, é uma pequena mosca preta de cerca de $2 \mathrm{~mm}$. ... Coletamos grande número dessas moscas, que foram dissecadas e examinadas à procura de parasitos, mas nenhuma estava parasitada. Todos os tripulantes e passageiros foram muito picados por essas moscas e parece-nos óbvio que elas não transmitem qualquer infecção (Strong et alii, 1926, p. 149).

A oncocercose foi detectada no Brasil em 1967, justamente em área próxima à região investigada em 1924 pela expedição Rice.

A elevada incidência da doença na África levou os países colonizadores a retomar os estudos sobre a oncocercose após a Primeira Guerra Mundial. A Organização de Saúde da Liga das Nações deu apoio à criação de institutos de pesquisa na região, à realização de expedições multinacionais em busca de vetores, à organização de conferências e à implementação de estratégias e profilaxia de erradicação da doença.

Concomitantemente, os médicos e cientistas latino-americanos manifestavam crescente preocupação com o avanço da doença no continente americano. Foram enviadas comissões a regiões endêmicas da África e investigadores europeus visitaram os focos emergentes no continente americano. Desde então diferentes hipóteses começaram a surgir, concernentes à origem da doença nas Américas, em conferências e debates. 


\section{Delimitação e hipóteses}

Segundo a teoria mais acatada sobre a origem da oncocercose, ela seria originária da África e teria sido introduzida nas Américas por meio do tráfico de escravos. A filária $O$. volvulus teria chegado primeiramente à Guatemala e atingido o México por efeito dos movimentos migratórios de indivíduos infectados. A importação de mão-de-obra africana teria sido responsável, também, pela introdução da doença na Colômbia, Venezuela e Brasil. Contudo, o mapeamento da doença nas Américas mostra focos completamente isolados uns dos outros, levando mesmo a especulações sobre uma possível origem autóctone da doença.

Considerando que várias doenças, entre elas a esquistossomose, foram introduzidas no Brasil através do tráfico de escravos, é plausível a hipótese de que escravos africanos portadores de Onchocerca levaram a doença à Guatemala, que seria o foco de passagem para o México, mas não existem ainda evidências para comprovar esta hipótese. Como observou Richard Strong (1934), não há nenhum registro da doença entre escravos recém-chegados à América, nem de qualquer invasão de escravos fugitivos para os centros endêmicos na Guatemala. Outra possibilidade levantada pelo pesquisador alemão Dampf (1931) é de que os índios guatemaltecos tenham adquirido a doença durante incursões à costa caribenha, que na época abrigava expressiva população negra. Em seu regresso, transplantaram a verminose para a região montanhosa onde ocorre hoje. Esta hipótese também carece de comprovação, já que não há registros da doença na costa caribenha onde eram desembarcados, em grande número, os escravos.

A hipótese de origem autóctone da oncocercose baseia-se unicamente em estudos arqueológicos de crânios pré-colombianos olmecas encontrados com perfurações semelhantes àquelas causadas por oncocercomas. Tampouco obtiveram confirmação as especulações sobre a introdução da doença no Novo Mundo através de algum reservatório animal autóctone ou gado infectado. A confirmação, na década de 1970, de que a doença grassava entre os ianomâmis, e tinha alto grau de endemicidade na região em que habitavam, reavivou questões até hoje não resolvidas. Como teria surgido a oncocercose em áreas tão remotas e isoladas umas das outras? Teria sido transportada por escravos levados por colonizadores espanhóis e portugueses que buscavam ouro no noroeste da Amazônia? Teria sido introduzida por viajantes recentes?

Os relatos dos naturalistas que visitaram a região registram somente as precaríssimas condições de saúde locais, com prevalência de moléstias como malária, reumatismo, diarréia, oftalmia e lepra. Não fazem nenhuma menção a sintomas que possam ser associados à oncocercose. De qualquer forma, uma revisão criteriosa destas narrativas, à luz dos conhecimentos atualmente disponíveis, poderia fornecer subsídios para a elucidação das questões relacionadas à distribuição da doença. 
Estudos recentes mostram que se encontra disseminada no continente africano, da costa Atlântica ao oceano Índico e ao mar Vermelho, e na península arábica, havendo registros de casos em Angola, Arábia Saudita, Benin, Burkina Fasso, Burundi, Camarões, Chade, Congo, Costa do Marfim, Etiópia, Gabão, Gâmbia, Gana, Guiné, Guiné-Bissau, Guiné Equatorial, Iêmen, Libéria, Malaui, Mali, Níger, Nigéria, Quênia, República Centro-Africana, Ruanda, Senegal, Serra Leoa, Sudão, Tanzânia, Togo, Uganda, Zâmbia, Zaire (OMS, 1995 apud Maia-Herzog, 1999).

A oncocercose é hoje caso de saúde pública, tanto na África como na Guatemala, e no México, onde é particularmente elevado o índice de cegueira causada pela doença. Programas de combate implementados na África pela Organização Mundial de Saúde (OMS) têm obtido relativo sucesso, reduzindo a incidência da doença em algumas áreas. Estudos recentes mostram, contudo, que ela vem aumentando em algumas regiões previamente controladas em virtude de migrações humanas (Maia-Herzog, 1999). No Brasil, um caso novo de oncocercose surgiu em Goiás, em 1986, em uma jovem de 16 anos que nunca havia deixado a região (Gerais e Ribeiro, 1986). Estudos em desenvolvimento têm tentado esclarecer de que forma se teria dado essa infecção. Teria sido por via da migração de garimpeiros ou de borrachudos infectados?

Acreditamos que somente uma abordagem multidisciplinar permitirá reconstruir a história da chegada e disseminação da oncocercose nas Américas. Nossa intenção é desenvolver um estudo comparativo sobre a disseminação da doença na África e na América Latina. Pretendemos obter dados sobre a introdução de escravos nas regiões afetadas pela oncocercoce. Analisaremos minuciosamente os relatos dos naturalistas e exploradores que percorreram as regiões hoje reconhecidas como endêmicas. Investigaremos a rede de relações estabelecida pelos cientistas latino-americanos, europeus e americanos que buscam entender a doença, e as campanhas de saúde desfechadas no combate a ela.

A meta final do projeto é um livro sobre a história da oncocercorse na América e a publicação de uma coletânea com artigos debatidos em um seminário.

\section{REFERÊNCIAS BIBLIOGRÁFICAS}

Baez, M. M. 1962

Bell, $\mathrm{H}$.

1999

Bertolli Filho, C. 2000

Blacklock, B. 1926
'Historia de la enfermedad de Robles (oncocercosis humana)'. Epoca V-Salud Públ., México, vol. IV(6): 915-38.

Frontiers of medicine in the Anglo Egyptian Sudan. Oxford, Oxford University Press.

História da saúde pública no Brasil. São Paulo, Ática.

'The further development of Onchocerca volvulus Leuckart in Simulium damnosum Tehob'. Ann. Trop. Med. Paras., 20: 203-18. 
Busvine, J. R. 1993

Cueto, $M$. 1977

Dampf, A. 1931

Ferreira, M. M. (org.) 1994

Fleck, L. 1981

Gerais, B. B. e

Ribeiro, T. C.

1986

Joly, P. R. 1898

Jordanova, L. J. 1979

Kiple, K. F. (org.) 1987

Kuhn, T. 2000

Leavitt, J. W. e Numbers, R. L. (orgs.) 1985

Lutz, A. 1909

Maia-Herzog, M. et alii 1999

Maia-Herzog, M. 1999

McNeill, W. H. 1976

Moraes, M. A. P. e Dias, L. B.

1973

Moraes, M. A. P. e Dias, L. B. 1972

Moraes, M. A. P. e Shelley, A. J. 1986
Disease transmission by insects. Its discovery and 90 years of effort to prevent it. Berlim/Heidelberg/Nova York, Springer-Verlag.

El regreso de las epidemias: salud y sociedad en el Perú del siglo XX. Lima, IEP.

'Simulidae vectors of the onchocerciasis in Oaxaca and Chiapas States'. Science, N. S. 74 (10), supplement.

História oral e multidisciplinaridade.

Rio de Janeiro, Diadorim Editora, pp. 45-73.

Genesis and development of a scientific fact. Chicago,

The University of Chicago Press.

'Relatos de casos - Oncocercose: primeiro caso autóctone da região centro-oeste do Brasil'. Rev. Soc. Bras. Med. Trop., vol. 19, no 2, pp. 105-7.

'Os insetos na transmissão das moléstias contagiosas'.

O Brazil-Medico, 20, 16, pp. 140

'Earth science and environmental medicine: the synthesis of the late enlightenment'. Em L. J. Jordanova e Roy Porter (orgs.), Images of the Earth: essays in the history of the environmental sciences. Londres, British Society for the History of Science.

The African exchange: towards a biological bistory. Durham/Londres, Duke University Press.

'What are scientific revolutions?'. Em Thomas Kuhn (org.), The road since structure: philosophical essays (1970-1993). Chicago, The University of Chicago Press, pp. 13-32.

Sickness and health in America - readings in the history of medicine and public health. Madison, The University of Wisconsin Press.

'Contribuição para o conhecimento das especies brazileiras do genero Simulium'. Memórias do Instituto Oswaldo Cruz, t. 1, no 2, pp. 1224-46.

'Discovery of a new focus of human onchocerciasis in central Brazil'.

Transactions of the Royal Society of Tropical Medicine and Hygiene, Londres, 93: 235-39.

'A oncocercose humana no Brasil e sua dispersão'. Tese de doutoramento em biologia parasitária, Rio de Janeiro, Escola Nacional de Saúde Pública, Instituto Oswaldo Cruz, Fiocruz.

Plagues and people.

Nova York, Doubleday.

'Onchocerciasis in Brazil'.

Bull. Pan Am. Health Organ., 7: 40-56.

'Oncocercose no estado do Amazonas, Brasil'.

Rev. Inst. Med. Trop. de São Paulo, 14: 330-3.

'Oncocercose no grupo Yanomama'. Em M. A. Ibáñez-Novion, A. M. Teixeira (orgs.) Adaptação à enfermidade e sua distribuição entre grupos indígenas da bacia Amazônica. Brasília, I, Centro de Estudos e Pesquisas Antropológicas e Médicas Polonoroeste, 112-19. 
OMS (Organização

Mundial de Saúde) 1995

Porter, D.

Ranger, T. e Slack, P. 1992

Rassi, B. E.; Monzón, H.; Castillo, M.;

Hernandez, I.;

Ramirez-Perez, J.

e Convit, J. 1977

Robles, R.

Rosemberg, C. E. 1995

Rosen, G. 1994

Sá, M. R. e

Maia-Herzog, $M$. jan.-fev.-mar. 2002

Sá, M. R.; Maia-Herzog,

M. e Shelley, A. J. 2001

Shelley, A. J. 1988

Strong, R.; Shattuck, G.; Bequaert, J. e Wheeler, $\mathrm{R}$. 1926

Strong, R. 1934

Thornton, E.

Warner, J. $\mathrm{H}$ 1995

Worboys, $M$. 1988
'Onchocerciasis and its control'. WHO Expert Commitee on Onchocerciasis Control. Technical Report Series, no 852.

Health, civilization and the State: a bistory of public bealth from ancient to modern times. Londres, Routledge.

Epidemics and ideas: essays on the bistorical perception of pestilence. Cambridge, Cambridge University Press.

'Discovery of a new onchocerciasis focus in Venezuela'.

Bull. Pan. Am. Health Org., 11: 41-64.

'Onchocercose humaine au Guatémala produisant la cécité et l'erysipele du 1919 littoral (erisipela de la costa)'. Bull. Soc. Path. Exot., 12 (7): 442-63.

Explaining epidemics and other studies in the bistory of medicine. Cambridge, Cambridge University Press.

Uma bistória da saúde pública.

São Paulo, Unesp/Hucitec/Abrasco.

'Oncocercose: um futuro caso de saúde pública no Brasil?'.

Inteligência, ano V, no 16, pp. 74-9.

'Tropical diseases and search for their carriers: vectors and parasites. The onchocerciasis history in Latin America and Africa'. XXI International Congress of History of Science, symposia, pp. 127-8.

'Of the vector aspects. Epidemiology of onchocerciasis in Latin America'. Ann. Review Entomol., 33: 337-66.

'Medical report of the Hamilton Rice seventh expedition to the Amazon in conjunction with the Department of Tropical Medicine of Harvard University, 1924-25'. Contributions from the Harvard Institute for Tropical Biology and Medicine, IV. Cambridge, Harvard University Press, p. 313.

'Onchocerciasis with special reference to the Central America form of the disease'. Em: J. H. Sandground, J. C. Bequaert e M. M. Ochoa, (orgs.), Onchocerciasis in Central America. Contributions from the Department of Tropical Medicine and the Institute for Tropical Biology and Medicine, VI, Cambridge, Harvard University Press, p. 234.

'Report of the Pan-African Health Conference. Johannesburg, Nov. 20-30, 1935'. Quartely Bulletin of the Health Organisation - League of Nations, vol. 5 , no 1 , pp. 1-209.

'The history of science and the sciences of medicine'. Em Arnold Thackray (org.), Constructing knowledge in the bistory of science. Osiris, vol. 10, pp. 164-93.

'Manson, Ross and colonial medical policy: tropical medicine in London and Liverpool, 1899-1914'. Em Macleod e Lewis (orgs.), Disease, medicine and empire: perspectives on Western medicine and the experience of European expansion. Londres, Routledge, pp. 21-38. 